\title{
Efficient Computation of Instantons for Multi- Dimensional Turbulent Flows with Large Scale Forcing
}

\author{
Tobias Grafke ${ }^{1, *}$, Rainer Grauer ${ }^{2}$, and Stephan Schindel ${ }^{2}$ \\ ${ }^{1}$ Department of Physics of Complex Systems, Weizmann Institute of Science, \\ Rehovot 76100, Israel. \\ ${ }^{2}$ Theoretische Physik I, Ruhr-Universität Bochum, Universitätsstr. 150, D44780 \\ Bochum (Germany).
}

\begin{abstract}
Extreme events play a crucial role in fluid turbulence. Inspired by methods from field theory, these extreme events, their evolution and probability can be computed with help of the instanton formalism as minimizers of a suitable action functional. Due to the high number of degrees of freedom in multi-dimensional fluid flows, traditional global minimization techniques quickly become prohibitive in their memory requirements. We outline a novel method for finding the minimizing trajectory in a wide class of problems that typically occurs in turbulence setups, where the underlying dynamical system is a non-gradient, non-linear partial differential equation, and the forcing is restricted to a limited length scale. We demonstrate the efficiency of the algorithm in terms of performance and memory by computing high resolution instanton field configurations corresponding to viscous shocks for 1D and 2D compressible flows.
\end{abstract}

PACS: 47.11.-j, 47.27.ef, 05.10.-a, 47.40.-x

Key words: instanton formalism, minimizing trajectory, numerical minimization, GPU programming, CUDA

\section{Introduction}

Systems in nature are almost always subject to noise. Even though these random perturbations often are small in amplitude, they nevertheless have drastic consequences on the behavior of the system as a whole by facilitating rare but extreme excursions of the dynamics. Many processes in biology, chemistry, physics and economics, including phase transitions [12], ocean dynamics [27], rates of chemical reactions [23], genetic switches [2] and option pricing in finance 3,5, are caused by rare extreme events.

\footnotetext{
${ }^{*}$ Corresponding author. Email address: tobias.grafke@weizmann.ac.il (T. Grafke)
} 
In the small noise limit, these extreme events are in fact very predictable. The arising most probable transition trajectory of the rare event in turn allows for predictions regarding both the evolution of the rare event and its probability. In the context of the Martin-Siggia-Rose/Jansen/de Dominicis formalism [8,22, 25] these field configurations are termed instantons. They correspond to the minimizers of the rate function in the Freidlin-Wentzell theory of large deviations $[9,14]$.

A number of numerical algorithms have been devised to compute these field configurations. Some of them, like the nudged elastic band method [19] or the string method [11, are only applicable to the important sub-class of gradient systems, while others, most notably the minimum action method [10] and variants thereof [21, 31], are able to find the instanton configuration for more general cases. All have in common that they solve the problem globally, by discretizing the trajectory along the physical time and applying global operations on its entirety. For PDE systems with an infinite number of degrees of freedom, in particular in higher dimensions, such as arising in turbulent fluids, the memory requirements of these algorithms quickly become prohibitive.

Many questions in fluid dynamics, such as shock formation in compressible flows [15] or the generation of rogue waves [26], allow an alternative to the global formulation due to the nature of their mixed initial/final boundary conditions. Here, it is feasible to iteratively solve the equations of motion (the instanton equations) of the underlying Hamiltonian system instead [7]. The boundary conditions are propagated throughout the domain, which opens up possibilities to avoid saving the field configuration at every instance in time. In particular, a drastic reduction in memory is possible by combining a number of techniques: (a) considering the "geometric" reparametrization of the trajectory instead of parametrization by physical time, (b) recursive storage of transition states, inspired by multigrid techniques, (c) exploiting the compactness of the support of the force correlation in turbulence setups, and (d) further memory reductions through wavelet compression. The detailed presentation of the resulting algorithm constitutes the core of this work. We illustrate its effectiveness by applying it to $1 \mathrm{D}$ and $2 \mathrm{D}$ compressible turbulence. In fact we demonstrate that the combined optimizations reduce the memory footprint enough to fit optimization problems with $N=10^{10}$ degrees of freedom on a single graphics card. It therefore becomes feasible to solve the numerical problem on graphics processing units (GPUs) instead of their host machines at a considerable gain in runtime performance. In consequence, all algorithms presented in this paper are implemented on GPUs using the CUDA framework [1. Furthermore, due to its memory efficiency, the scheme in principle allows attacking the important problem of the computation of instanton configurations for the 3D incompressible Navier-Stokes equation, which would yield scaling predictions for turbulent statistics.

This paper is organized as follows: We first establish the instanton formalism and the associated minimization problem in section/2. We introduce the Martin-Siggia-Rose/Janssen/de Dominicis formalism and present a beneficial modification of the action functional in terms of a geometric reparametrization. In section 3 , we outline the composition of our proposed algorithm. This includes a discussion of the recursive integration of the mixed initial/final 
value problem posed by the instanton equations and its combination with the projection of the auxiliary field variable. Section 4 presents applications of the proposed algorithm to the problem of prototypical shock configurations of one- and two-dimensional compressible fluids. We demonstrate the efficiency in memory of the algorithm by computing the minimizer in a high degrees of freedom setup and discuss the computational overhead of the optimizations in terms of performance. We conclude in section 5 with a discussion of the results, present possible future applications as well as comment on their potential impact on the understanding of turbulent fluids.

\section{Instantons formalism for stochastic partial differential equa- tions}

Consider a system of $n$ partial differential equations driven by a Gaussian noise,

$$
\dot{u}=b[u]+\eta(x, t)
$$

in $d$-dimensional domain, i.e. the field $u$ is a function $u(x, t): \mathbb{R}^{d} \times[-T, 0] \rightarrow \mathbb{R}^{n}$. The drift $b[u]$ is some possibly nonlinear operator and the Gaussian noise $\eta$ has a finite correlation length $L$ in space and is white-in-time,

$$
\langle\eta(x, t) \eta(x+r, t+s)\rangle=\chi(r) \delta(s) .
$$

We restrict ourselves to the case of a correlation function $\chi(r)$ with compact support in Fourier space,

$$
\hat{\chi}(k)=f(k) \theta\left(k_{c}-|k|\right),
$$

for an arbitrary shape $f(k), \theta$ denoting the Heaviside step function. Thus, the driving occurs only at scales larger than a cut-off scale $L \sim 1 / k_{c}$. This setup appears naturally in turbulent fluid systems with a direct cascade, such as 3D incompressible Navier-Stokes turbulence, MHD turbulence or Burgers turbulence: Energy is inserted at large scales and transported to small scales by the non-linear term, where it is dissipated by viscosity. Note that the opposite choice of small scale forcing, which is necessary for configurations with an inverse cascade, is equally viable despite being not of the form (2.3), as the only necessary condition is a compact support of the forcing correlation.

The Martin-Siggia-Rose/Janssen/de Dominicis path integral formalism allows us to formally write down the expectation of any observable of the field variables, $\mathcal{O}[u]$, under the noise $\eta$ as

$$
\langle\mathcal{O}[u]\rangle_{\eta} \propto \int D u \int D(i p) \mathcal{O}[u] \exp \left(-I_{T}[u, p]\right)
$$

with the action functional or response functional

$$
I_{T}[u, p]=\int_{-T}^{0}\left(\langle p, \dot{u}-b[u]\rangle-\frac{1}{2}\langle p, \chi p\rangle\right) d t,
$$


introducing the auxiliary field $p$. Here, $\langle\cdot, \cdot\rangle$ is the $L_{2}\left(\mathbb{R}^{n}\right)$-scalar product. Depending on the exact form of the observable at hand, it is then possible to estimate the expectation by approximating the path integral (2.4). For the case of transition possibilities between two known states $u_{-}$and $u_{+}$, this is usually done by a saddle point approximation, which amounts to finding the minimizers of the action functional (2.5), which are also termed instantons and correspond to the classical trajectories under the action functional $I_{T}[u, p]$. In fact, in the limit of extremely rare events, the saddle point approximation becomes exact, as rigorously derived in the Freidlin-Wentzell theory of large deviations [14]. To numerically find the minimizer of (2.5), one discretizes the action and subsequently employs global minimization techniques to solve the variational problem

$$
\inf _{T>0} \inf _{u} I_{T}[u, p]
$$

with appropriate boundary conditions $u(x, t=-T)=u_{-}(x), u(x, t=0)=u_{+}(x)$ (the auxiliary field is usually computed explicitly as $p=\chi^{-1}(\dot{u}-b[u])$ in this setup). This approach is commonly taken in practice, most notably by the string method 11 for gradient systems, and by the minimum action method [10] and variants thereof [28, 31] in more general setups.

For a wide class of questions the form of the observable $\mathcal{O}[u]$ allows for an efficient alternative approach: Suppose we only want to measure the observable at the final time $t=0$ (or any other distinct point in time), and furthermore only measure a single degree of freedom in the final field configuration. This could, for example, be the ocean surface elevation at a single point in a rogue wave setup, or an extreme velocity gradient for Burgers shock formation (see below). Note that in contrast to the computation of transition probabilities, the exact form of the final condition of the field $u$ is not prescribed, but instead is recovered as part of the solution. This allows to recover the exact form in space and time of the extreme ocean surface wave form or extreme shock structure, respectively, from the formalism. In the outlined setup, the observable takes the form

$$
\mathcal{O}[u]=\delta(F(u(x, t=0))-a)
$$

for a scalar functional $F(u)$. For example, we choose $F(u)=u_{x} \delta(x)$ to measure the probability of a gradient $a$ in the origin, or $F(u)=u \delta(x)$ to measure a surface elevation of $a$ in the origin. In general, we can rewrite this observable as a Fourier integral,

$$
\mathcal{O}[u]=\delta(F(u(x, t=0))-a)=\int d(i \lambda) \exp \left(\int_{-T}^{0} \lambda(F[u]-a) \delta(t)\right)
$$

and insert it into the path integral formulation (2.4),

$$
\langle\mathcal{O}[u]\rangle_{\eta} \propto \int D u \int D(i p) \int d(i \lambda) \exp [-\underbrace{\left(I_{T}[u, p]-\lambda(F[u]-a)\right)}_{S_{T}[u, p]}],
$$


where we label the modified action functional in the exponent of the path integral as $S_{T}[u, p]$. Similar to the case of transition probabilities, we want to estimate the expectation by employing a saddle point approximation and computing the instanton field configuration. Instead of globally minimizing the action functional we resort to solving the equations of motion or instanton equations for the associated Hamiltonian system instead 7,15$]$. These follow from the realization that at the saddle point the variation of the functional $S_{T}[u, p]$ with respect to the fields $u, p$ vanishes, leading to

$$
\begin{aligned}
& \dot{u}=b[u]+\chi p \\
& \dot{p}=-(\nabla b[u])^{T} p+\lambda \nabla F[u] \delta(t),
\end{aligned}
$$

where $\nabla$ is meant in the functional sense with respect to the field variable. Now, the second term of the auxiliary equation, $\lambda \nabla F[u] \delta(t)$, which incorporates the observable, acts only on the final time $t=0$. It can therefore be understood as a final condition for the auxiliary field [4, 18]. On the other hand, the final configuration of the field $u$ is not known. For this reason, global minimization techniques are harder to implement in this case. Though in principle a modification of the variational formulation $(2.6)$ is possible in terms of e.g. penalty methods which enforce the constraint on the final field configuration, integrating the equations of motions is a much more natural approach: The boundary constraints are automatically fulfilled when propagating the initial configuration of $u$ forward in time via equation (2.8a), while propagating the final conditions of $p$ backwards in time via equation (2.8b). The final field configuration $u(x, t=0)$ adheres the constraints of the observable and at the same time yields the most probable composition of the unconstrained degrees of freedom. Note also, that equation (2.8a) resembles the original stochastic system (2.1), with the random noise $\eta$ being replaced by the convolution $\chi p$. A solution of the instanton equations $(2.8)$ therefore not only allows for a prediction of the evolution of the field leading up to the extreme event at $t=0$, but additionally yields the corresponding force.

\subsection{Geometric action functional and arc-length reparametrization}

We want to consider the case $T \rightarrow \infty$, i.e. the infinite time minimizer. This case arises in general when asking for the emergence of an extreme event out of a stationary state, which in terms of large deviations corresponds to a diffusive exit from a stable fixed point of the unperturbed dynamics. From a numerical point of view, the limit $T \rightarrow \infty$ poses the obvious problem of how to discretize an infinite time interval appropriately.

For minimization problems of the form (2.6), the form of the instanton configuration is independent of the parametrization of the trajectory. It is therefore possible to reformulate the variational problem into a minimization on the space of arc-length parametrized curves instead. The modified action functional,

$$
I[u, \dot{u}]=\int_{0}^{1}\left(\|\dot{u}\|_{\chi}\|b[u]\|_{\chi}-\langle\langle\dot{u}, b[u]\rangle\rangle_{\chi}\right) d s,
$$


is shown to be independent of the parametrization [21], such that the search space is restricted to trajectories of unit length. The metric is induced by the correlation matrix $\chi$ of the problem via

$$
\left\langle\langle u, v\rangle_{\chi} \equiv\left\langle u, \mathcal{F}^{-1}\left(\hat{\chi}^{-1} \hat{v}\right)\right\rangle_{L^{2}},\right.
$$

where $\mathcal{F}$ denotes the Fourier transform operator and $\hat{f} \equiv \mathcal{F}(f)$ and the norm $\|\cdot\|_{\chi}$ is defined accordingly. The action functional (2.9), also termed the geometric action functional, was considered in 17, 21,29 as foundation of a global minimization technique, the geometric minimum action method (gMAM).

In the context of this work, it is used to derive the corresponding geometric equations of motion:

$$
\begin{aligned}
\dot{u} & =\frac{\|\dot{u}\|_{\chi}}{\|b[u]\|_{\chi}}(b[u]+\chi \star p) \\
\dot{p} & =-\frac{\|\dot{u}\|_{\chi}}{\|b[u]\|_{\chi}}\left((\nabla b[u])^{T} p+\lambda \nabla F[u] \delta(t)\right) .
\end{aligned}
$$

With this set of equations of motion it is possible to numerically discretize the instanton configuration with a finite number of grid points even if the trajectory is traversed in infinite physical time. Furthermore, the arc-length parametrization, using the correlation as metric for the phase space, naturally improves the numerical resolution in phases with critical dynamics, as it automatically adapts to the evolution of the action density.

\section{$3 \quad$ Numerical solution of the instanton equations}

Above, the problem of the computation of instanton configurations has been narrowed down to numerically finding the solution to the instanton equations (2.8) or (2.11) in the case of $T \rightarrow \infty$, together with an initial condition for the field $u(x,-T)=u_{\text {start }}(x)$ and a final condition for the auxiliary field of the form $p(x, 0)=p_{\text {end }}(x)=-\lambda \nabla F[u]$. As the field equation and the auxiliary equation are mutually dependent, they cannot be solved without knowledge of the solution of other equation. Therefore, an iterative solution of the instanton equations was proposed in 7 . For any approximation $u_{k}(x, t)$ of the field variable, we can solve the auxiliary equation (2.11b backwards in time, starting the integration with from the final condition $p_{\text {end }}(x)$, to obtain an approximation $p_{k}(x, t)$. We then use this approximation to solve the field equation (2.11a) forward in time, starting from its initial condition $u_{\text {start }}(x)$, to obtain the approximation $u_{k+1}(x, t)$ for the field variable. The iteration is started with an initial guess for the field variable $u_{0}(x, t)$ (e.g. $\left.u_{0}(x, t)=0\right)$. We iterate until a fixed point is reached, at which point $\left(u_{k}(x, t), p_{k}(x, t)\right)$ are a solution to the instanton equations.

First, note that this scheme requires the storage of the field variable and the auxiliary variable for all times. This issue will be addressed below. Furthermore, the instanton equations are formally very similar to the original noisy system (2.1). The numerical solution can therefore be accomplished with a similar set of tools as the solution of the 
underlying fluid equation. In particular, for the field equation it is possible to reuse existing codes that solve a stochastically forced fluid system and simply replace the random force with the deterministic term $\chi \star p$. The auxiliary field, as visible in the example applications below, usually has a very similar structure. This is in contrast to the variational approach, which requires the development of a new toolset to numerically minimize the action functional.

\subsection{Recursive solution of the mixed initial/final value problem}

The most obvious challenge in computing the instanton in the above laid out fashion for large systems is the requirements in memory. Both for global minimization techniques and for iterative solutions of the equations of motion, the field configuration has to be computed and saved for every instance in time, which basically increases the problem dimension by one. For a class of related problems, an effective algorithm is proposed in [6] which scales as $\mathcal{O}\left(\log N_{t}\right)$ in memory consumption instead of $\mathcal{O}\left(N_{t}\right)$, with only a logarithmic increase in computational cost. In this section, we present the extension of this algorithm to the class of mutually dependent initial/final value problems in the case of instanton equations with large scale force correlations. Note that similar ideas are known in the field of PDE constrained optimization as "checkpointing" 30.

The instanton equations (2.11) represent a mixed initial/final value problem: Half of the unknowns are given an initial value and propagated forward in time, while the others are given a final value and are propagated backwards in time. These conditions are also encountered in other physical contexts, e.g. the transport of a passive scalar by an evolving flow with a given final density, as described in $[6]$. Consider a simpler system of equations of the form

$$
\begin{array}{rrr}
u_{t}=f(u, t), & u\left(t_{1}\right)=u_{1} \\
p_{t}=g(u, p, t), & & p\left(t_{2}\right)=p_{2}
\end{array}
$$

with $t_{1}<t_{2}$, as considered in [6]. The naive way to solve the system (3.1) would be to first solve equation (3.1a) forward in time, starting with the initial condition $u_{1}$ at $t_{1}$, while saving the complete evolution of $u(t)$ along the way, and subsequently solving equation (3.1b) backward in time, starting from $p_{2}$ at $t_{2}$, using the previous solution $u(t)$ to evaluate $g(u, p, t)$ along the way. This approach is $\mathcal{O}\left(N_{t}\right)$ in both memory and computing time. It represents the worst case in memory usage (every time-step is saved) and the optimal case in computation time (every time-step is only computed once). The other extreme would be to only compute equation (3.1b) backwards in time, starting from $p_{2}$ at $t_{2}$, and computing the needed $u(t)$ at each instance in time by integrating equation (3.1a) from $t_{1}$ to $t$. Here, the memory cost is $\mathcal{O}(1)$, while the computational cost scales like $\mathcal{O}\left(N_{t}^{2}\right)$. Of course, this variant is only possible due to the fact that equation (3.1a) is independent of the field $p$. In the case of mutual dependence, such as the system of instanton equations 2.11), this variant does not apply. 


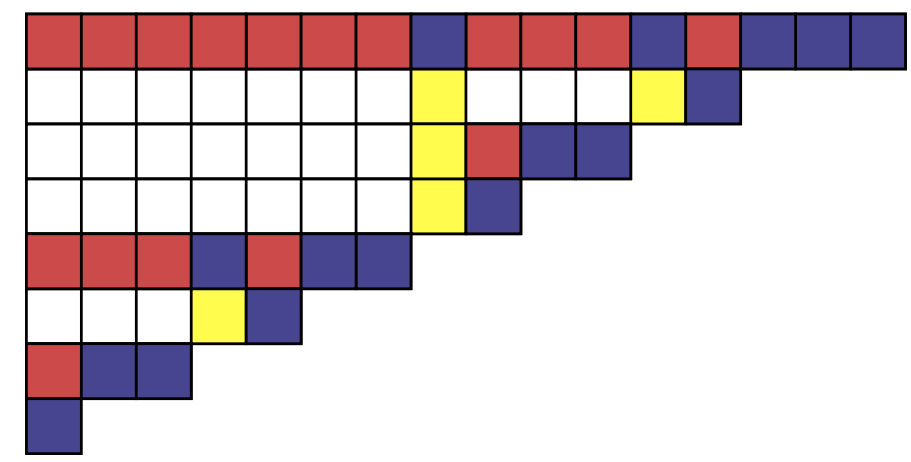

Figure 1: Depiction of the recursive integration algorithm for $k=2$. Each square represents one of the 16 timesteps, showing steps computed but subsequently dropped (red), computed and saved to memory (blue/dark) and loaded from memory (yellow/light). Total memory requirement is the maximum number of green and blue boxes in a line $\left(5\right.$ in this example), which is $\mathcal{O}\left(\log N_{t}\right)$. Total computation time is the total number of red and green boxes (33 in this example), which is $\mathcal{O}\left(N_{t} \log N_{t}\right)$.

A sensible compromise between memory and computing time can be achieved when realizing that both above algorithms can be used as building blocks in the following sense: Divide the interval $\left[t_{1}, t_{2}\right]$ into $k$ sub-intervals. Compute the solution of equation (3.1a) forward in time from $t_{1}$ to $t_{2}$, saving $u(t)$ along the way only at the start of each of the $k$ intervals. Now, it is possible to solve the original problem on each sub-interval of size $N_{t} / k$ with either of the two algorithms from above. Note that this modification merely changes the constants, but does not modify the scaling behavior of memory and computing time.

Finally, since for each sub-interval the problem in structure resembles the original problem, the division outlined above can be recursively repeated, until the interval size corresponds to the time discretization $\delta t$. At this point, no further solution inside the subinterval is necessary, since the interval represents only one integration step. As inspired by similar principles in multi-grid algorithms or the fast Fourier-transform, a natural choice is $k=2$. Then, the memory requirement scales as $\mathcal{O}\left(\log N_{t}\right)$ and computing time as $\mathcal{O}\left(N_{t} \log N_{t}\right)$. A schematic depiction of the algorithm for $k=2$ is shown in Figure 1 . For the initial solution of the field equation, the field is stored at the intermediate timesteps $i \epsilon$ $\{8,12,14,15,16\}$, of which the last 3 can immediately be used for the backwards propagating auxiliary equation. Whenever a timestep is encountered for which the field configuration is not stored, it is propagated forward again from the last known position, while storing intermediate values again in the fashion lined out above. The choice $k=2$ proves to be a good compromise between memory and computing time, even though any $k$ is admissible to use the available memory at optimal efficiency (even fractional $k$ are possible, choosing the interval boundaries at $\left.\left.t_{i}=t_{1}+\frac{t_{2}-t_{1}}{k} i, i \in\{1, \ldots, \mid k\rfloor\right\}\right)$. In the following section, we will apply this algorithm to instanton equations of the form (2.11) by taking advantage of the form of the forcing correlation (2.3). 


\subsection{Projecting the auxiliary field}

In fact, the instanton equations (2.11) are not of the form of equation (3.1): Equations 2.11a and 2.11b mutually depend on each other, prohibiting the purely recursive form lined out above, because neither $u$ nor $p$ can be integrated without knowledge of the other field. It is therefore necessary to store at least one field completely. Yet, the dependence of the $u$-equation on the $p$ is solely through the term $\chi \star p$, which, due to its compact support in Fourier space (equation (2.3)), acts only on few modes. The convolution of the auxiliary field with the forcing correlation can therefore be seen as a projection on the active modes of the forcing.

Consequently it is advantageous, instead of saving any of the field configurations $u$ or $p$, to only store the projection, i.e. the non-vanishing modes of the field $\chi \star p$ for all time-steps. From this one can reconstruct the velocity field $u$ in the recursive manner lined out above. This approach does not recover the $\mathcal{O}\left(\log N_{t}\right)$ scaling in memory, but restricts the number of degrees of freedom in every other dimension, therefore preserving advantageous memory requirements. More importantly, since the physical cut-off scale $k_{c}$ is a constant, the memory cost of the auxiliary field becomes independent of the number of degrees of freedom in space $N_{x}$.

Also note that in some applications it is actually feasible to integrate the whole auxiliary equation (2.11b) in the convoluted field variable instead. Even though this approach does not reduces the number of active modes (as mixing of modes is possible through the nonlinear drift term), it provides a beneficial mollification of the auxiliary variable due to the large-scale nature of the correlation function $\chi(x)$.

\section{Applications}

We now present several applications of the proposed algorithm. Every integration of the instanton equations (2.11) is done with a second order Runge-Kutta integrator in time. Spatial derivatives are computed with a pseudo-spectral scheme using fast Fourier transforms (FFT). Due to the high memory efficiency of the presented algorithm a speedup of the computation using graphic cards becomes feasible. All computations presented hereafter where conducted on a single graphics card using the CUDA toolkit 1].

We want to focus on the Burgers equation,

$$
\partial_{t} \mathbf{u}+\mathbf{u} \cdot \nabla \mathbf{u}-\nu \Delta \mathbf{u}=\mathbf{f}
$$

in one or two dimensions, $\mathbf{u}(x, y, t): \mathbb{R}^{d} \times[-T, 0] \rightarrow \mathbb{R}^{d}, d=1$ or $d=2$, with force correlation

$$
\left\langle f_{i}(\mathbf{x}+\mathbf{r}, s+t) f_{j}(\mathbf{x}, s)\right\rangle=\delta(t) \chi_{i j}(r)
$$

of the form (2.2). For this system we want to solve the instanton equations (2.11) numerically. As observable we choose the gradient of the velocity field $\mathbf{u}$ in the origin for $d=1$, or a sensible generalization thereof for $d=2$, in order to find the most probable evolution of an extreme gradient event out of a stationary turbulent Burgers flow. 


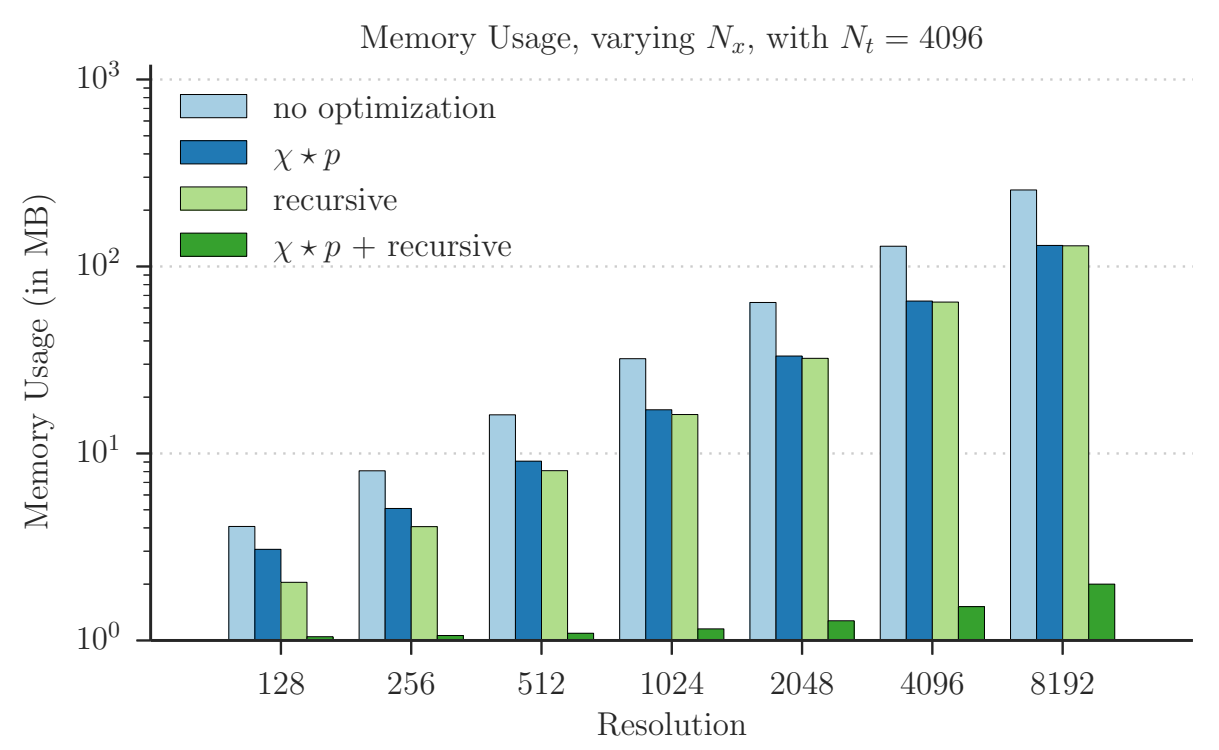

Figure 2: Comparison of memory costs for 1D simulations with varying spatial resolution and a fixed time resolution of $N_{t}=4096$. Using only either the recursive algorithm or the compactness of the forcing (denoted as " $\left.\chi \star p^{\prime \prime}\right)$ roughly halves the memory usage. Combining both methods leads to an improvement of memory efficiency of more than a factor $10^{2}$ (257MB naive vs. $2 \mathrm{MB}$ optimized).

\subsection{One dimensional Burgers equation}

For the 1D case, let the correlation in space $\chi(r)$ be of the form 2.3 with the shape being a Mexican hat, $f(k)=-k^{2} \exp \left(-k^{2} / 2\right)$. As observable we choose

$$
F[u]=\partial_{x} u(0,0),
$$

which conditions on the gradient of the velocity field in the space-time origin. This amounts to finding the most probable evolution of the 1D Burgers equation towards a shock with gradient $a$ at $x=0$ as a final configuration.

The physical implications of this choice are discussed in [16]. In particular, the computation of the instanton for the observable 4.3 allows the computation of the shape of the far left tail for the velocity gradient probability distribution function. In addition to being a computational tool, the instanton configuration was furthermore demonstrated to correspond to the most extreme events in turbulent Burgers flows [15]. In particular, it was shown that the instanton configuration can be extracted directly from a stochastically driven Burgers flow, and corresponds in shape and evolution to the prediction.

Here, we focus on the computational efficiency of the proposed algorithm. For memory usage statistics, see figure 2. Note that both the projection of the forcing onto active modes and the recursive time integration each have only little effect on the memory consumption on their own. Only the combination of both optimizations leads to a drastic reduction in memory usage of about a factor 100. In particular, for the highest resolution that was 

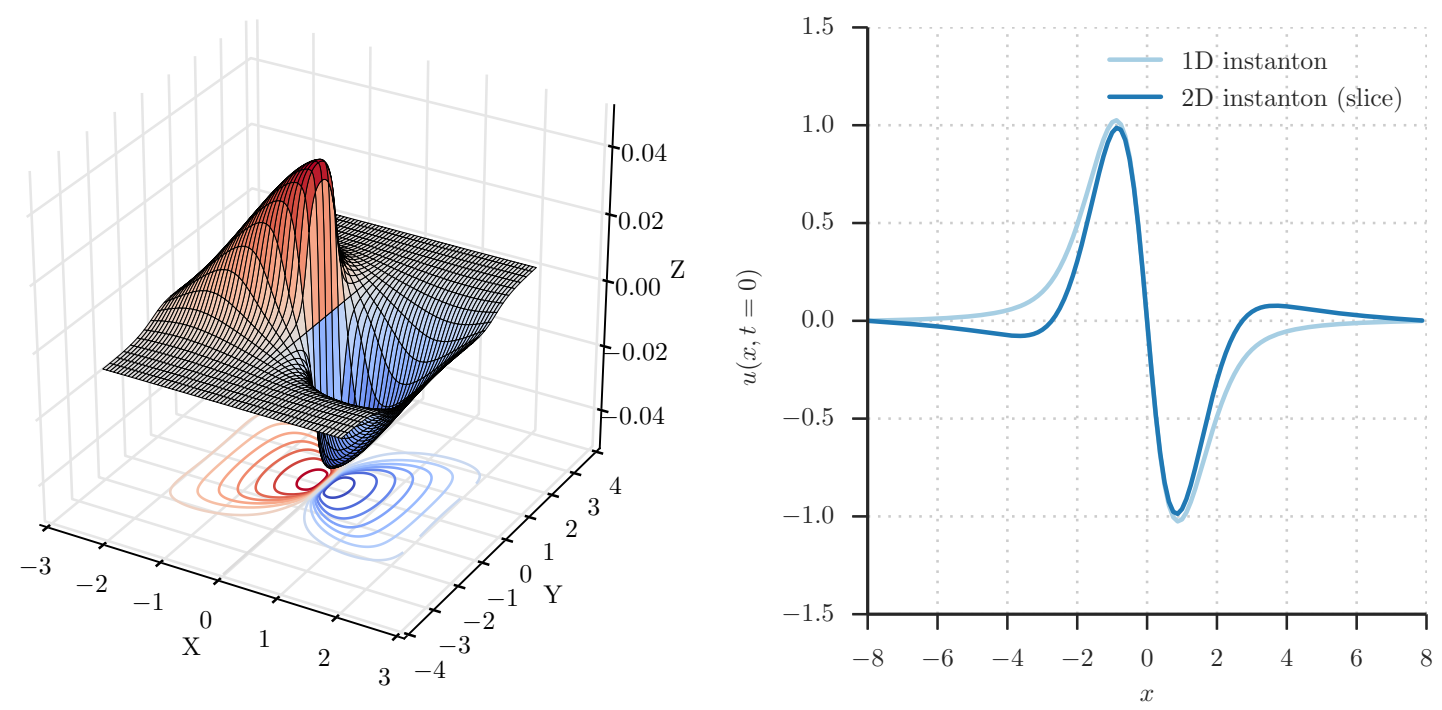

Figure 3: Left: Surface plot of the $x$-component of the velocity field, $u_{x}(x, y)$, for the 2D shock instanton configuration for the gradient $\partial_{x} u_{x}=-1, \nu=10^{-2}$. Right: Comparison between the 1D shock instanton configuration and a slice through the $2 \mathrm{D}$ shock instanton configuration for the gradient $\partial_{x} u_{x}=-2, \nu=\frac{1}{2}$.

tested, only $2 \mathrm{MB}$ of memory were required, in comparison to $257 \mathrm{MB}$ of the unoptimized version. On the other hand, due to the performance scaling with $\mathcal{O}\left(N_{t} \log N_{t}\right)$, the optimizations impose a computational cost of merely a factor 3 compared to the algorithm without any optimizations, which is small in comparison to the achieved memory savings.

\subsection{Two dimensional Burgers equation}

The 2D Burgers equation preserves irrotationality of the flow under corresponding forcing. Therefore, it is natural to decompose the forcing correlation function according to

$$
\chi_{i j}(r)=\alpha \chi_{i j}^{\mathrm{irr}}(r)+(1-\alpha) \chi_{i j}^{\mathrm{sol}}(r),
$$

where $\chi_{i j}^{\mathrm{irr}}$ and $\chi_{i j}^{\mathrm{sol}}$ denote irrotational and solenoidal forcing respectively. In two dimensions, they are realized through

$$
\begin{aligned}
& \chi_{i j}^{\mathrm{irr}}(r)=g(r) \delta_{i j}+r g^{\prime}(r) \frac{r_{i} r_{j}}{r^{2}} \\
& \chi_{i j}^{\mathrm{sol}}(r)=f(r) \delta_{i j}+\frac{r f^{\prime}(r)}{d-1}\left(\delta_{i j}-\frac{r_{i} r_{j}}{r^{2}}\right) .
\end{aligned}
$$

Note that the irrotational forcing is independent of the spatial dimension $d$, whereas the solenoidal forcing explicitly contains $d-1$ in the denominator of the second term.

In one dimension, only the irrotational forcing is allowed and reduces to

$$
\chi_{11}^{\operatorname{irr}}(r)=g(r)+r g^{\prime}(r) .
$$




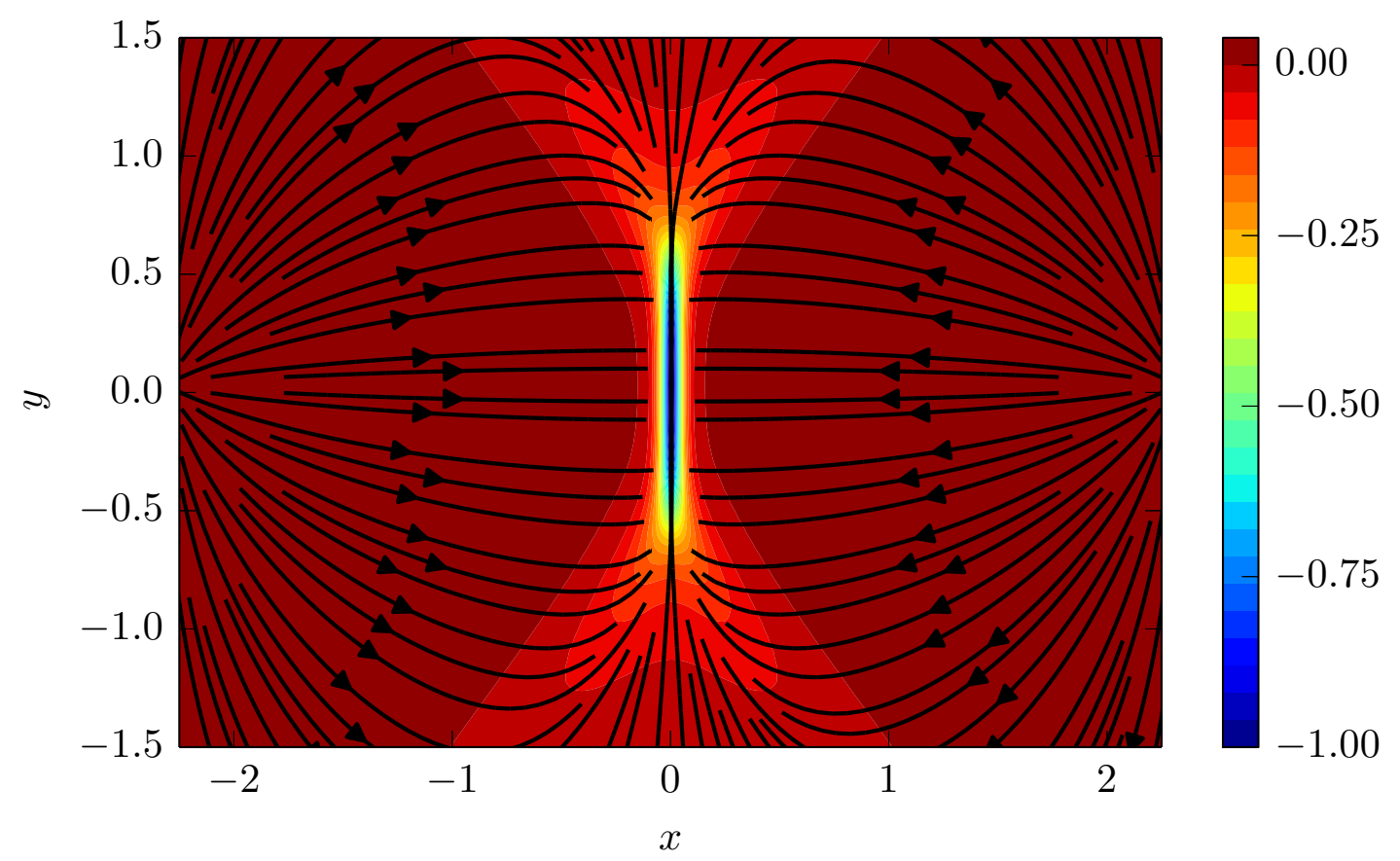

Figure 4: Contour plot of the velocity gradient in $x$-direction, $\partial_{x} u_{x}$ for the 2D instanton configuration for a gradient $\partial_{x} u_{x}=-1$ at $t=0$. The arrows depict the direction of the velocity field $u(x, y)$.

In the relevant case of large scale forcing, we require up to the cut-off scale that

$$
g(r)+r g^{\prime}(r)=-\partial_{r r} e^{-\frac{r^{2}}{2}}
$$

to match the $1 \mathrm{D}$ case, which directly leads to

$$
g(r)=e^{-\frac{r^{2}}{2}}
$$

We will restrict ourselves to purely irrotational forcing, $\alpha=1$, in the following. The instanton equations 2.8 for the 2D Burgers equation read

$$
\begin{aligned}
\partial_{t} \mathbf{u}+\mathbf{u} \cdot \nabla \mathbf{u}-\nu \Delta \mathbf{u}=\chi \star \mathbf{p} \\
\partial_{t} \mathbf{p}+\mathbf{u} \cdot \nabla \mathbf{p}-(\mathbf{p} \times \nabla) \mathbf{u}^{\perp}+\nu \Delta \mathbf{p}=0,
\end{aligned}
$$

with $\mathbf{u}^{\perp}=\left(-u_{y}, u_{x}\right)$ and $(\chi \star \mathbf{p})_{i}=\sum_{j} \chi_{i j} \star p_{j}$. There are several choices to generalize the observable (4.3) to more than one space dimension: Setting $F[\mathbf{u}]=\nabla \cdot \mathbf{u}(0,0)$ selects configurations of extreme velocity divergence in the origin, which amounts to compressible "explosions" for $a>0$ and "implosions" for $a<0$. Here, we will focus on the choice $F[\mathbf{u}]=\partial_{x} u_{x}(0,0)$. This choice corresponds to high gradients in $x$-direction, resulting in prototypical $2 \mathrm{D}$ shock configurations that dominate the statistics of turbulent 2D Burgers flows. 

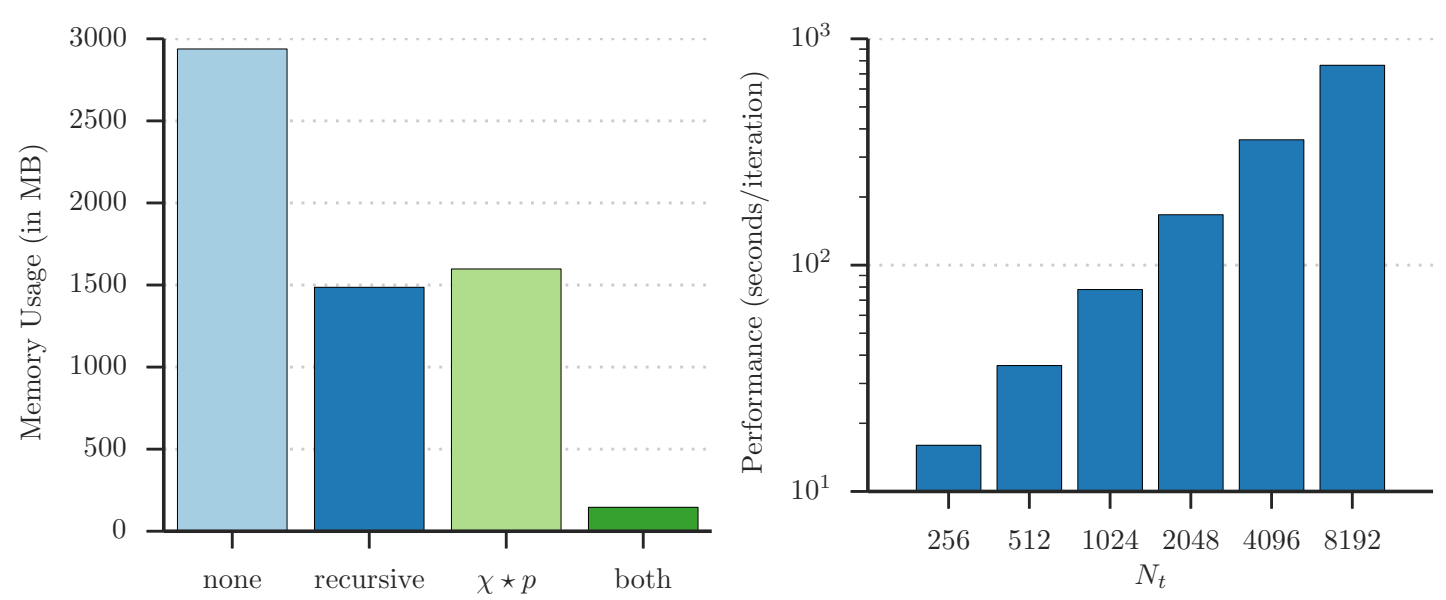

Figure 5: Left: Comparison of memory costs for 2D simulations with $N_{x}=256 \times 256, N_{t}=2048$ for the presented optimizations. The total memory saving of the combined algorithm exceeds a factor of 20 . Right: Performance of the optimized algorithm for $N_{x}=1024 \times 1024$ and varying $N_{t}$ scales as $\mathcal{O}\left(N_{t} \log N_{t}\right)$.

The shock structure for a gradient $\partial_{x} u_{x}(0,0)=-1$ is depicted in the surface plot of the $x$-component of the velocity field $\mathbf{u}(x, y)$ at the final time $t=0$ in figure 3 (left). This configuration constitutes the most probable shock structure with the chosen gradient in the limit of small forcing. A comparison between the 1D instanton configuration and a cut through the $2 \mathrm{D}$ instanton configuration perpendicular to the shock at $y=0$ is presented in figure 3 (right). Note that the higher-dimensional setup cannot reduce to a completely one-dimensional structure independent of $y$, since the assumption of isotropy of the forcing, in combination with the optimal forcing in $x$-direction, leads to a residual non-vanishing velocity component $u_{y}$. The size of the shock structure along the shock is therefore determined by the correlation length of the forcing $L=1$, while its size perpendicular to the shock is determined by the viscosity $\nu \ll 1$. This is clearly visible in figure 4 , where the contour plot shows the $x$-component of the gradient in $x$-direction, $\partial_{x} u_{x}$, and the arrows depict the direction of the velocity field $\mathbf{u}(x, y)$ with $N_{x}=2048 \times 512, N_{t}=2048$ and $\nu=0.01$.

The highest resolution achieved is $N_{x}=1024 \times 1024, N_{t}=8192$, with a total memory usage of $577 \mathrm{MB}$, performance of 764s/iter on a desktop computer, Intel Xeon E5-1620 (3.6GHz), GeForce GTX680 (GK104 "Kepler"). This amounts to a number of degrees of freedom of $N=N_{x} \times N_{t}=2^{33} \approx 10^{10}$ on a single graphics card, which is about a factor $10^{5}$ more than state-of-the-art global minimization algorithms in $2 \mathrm{D}$ settings (e.g. nucleation in the presence of shear with $N_{x}=64^{2}, N_{t}=100$ [20], minimizers of the KPZ-equation with $N_{x}=100^{2}, N_{t}=100$ 13, or geophysical bi-stability for the quasi-geostrophic equations, $\left.N_{x}=16^{2}, N_{t}=200[24]\right)$. The extrapolated memory usage of the unoptimized algorithm is $183 \mathrm{~GB}$, which would exceed our implementation by more than a factor 300 , while the computational overhead of the combined optimization is again below a mere factor 3 in 
the total computation time. As the scaling in memory is sub-linear, it is to be expected that state-of-the-art hardware will allow for a disproportionally higher number of degrees of freedom to be computed. Figure 5 (left) shows a comparison of the memory cost for a lower resolution setup of $N_{x}=256 \times 256, N_{t}=2048$ in order to fit the unoptimized case on the machine: Both the recursive optimization and the projection method amount to a saving of roughly a half, the combination of both leads to memory savings of a factor 20. On the other hand, the computational performance overhead of the optimizations scales with $N_{t}$ as $\mathcal{O}\left(N_{t} \log N_{t}\right)$ even for large spatial resolution. This is depicted in figure 5 (right) for the fully optimized algorithm for $N_{x}=1024 \times 1024$ and varying $N_{t}$. Because of memory restrictions, a comparison to the unoptimized variant is only possible for the lowest value of $N_{t}=256$ with $7.9 \mathrm{~s} /$ iter (unoptimized memory usage) versus $16 \mathrm{~s} /$ iter (optimized memory usage).

\section{Conclusion and discussion}

We present a novel method for computing minimizers of the action functional arising in the context of the instanton formalism for the computation of extreme events for stochastic partial differential equations. In particular, it is applicable in the setup of multidimensional fluid equations with a direct energy cascade, where the energy is injected on large scales.

The algorithm combines several ideas from other fields to optimize the memory footprint of the minimization problem at a high number of degrees of freedom in both temporal and spatial direction at marginal computational overhead: In the spirit of [7, mixed initial/final boundary conditions of the equations of motion are integrated in time instead of solving the global optimization problem. Disadvantageous time-discretization in the presence of an infinite time minimizer is mitigated by employing the geometric action functional [17, 21] instead of the classical Martin-Siggia-Rose/Janssen/de Dominicis response functional. In time direction, we then extent a multigrid-inspired recursive time integration technique [6] to the setting of mutually dependent instanton equations. This is only effective in combination with a projection of the auxiliary field on its active modes, which is made possible by the large-scale correlation in space of the driving force in the turbulent regime. These optimizations amount to a $\mathcal{O}\left(\log N_{t}\right)$ dependence in memory of the field variable, while the memory usage of the auxiliary field becomes decoupled from the spatial resolution $N_{x}$. The computational effectiveness of the algorithm is demonstrated in the case of one- and two-dimensional compressible turbulence for the Burgers equation. We determine and compare the instanton configuration for a gradient observable in the $1 \mathrm{D}$ and $2 \mathrm{D}$ case, which amounts to computing the prototypical shock evolution and final configuration with the imposed gradient value.

Additionally, we demonstrate the ability to compute minimizing trajectories with as much as $N \approx 10^{10}$ degrees of freedom. The algorithm is therefore suitable to numerically determine the instanton field configuration for two- and three-dimensional incompressible 
turbulence that is conjectured to have significant impact on the evolution of turbulent fluids and dominate the tails of turbulent statistics.

\section{Acknowledgments}

The work of T.G. was partially supported through the grants ISF-7101800401 and Minerva Coop 7114170101. R.G.and S.S. acknowledge support through DFG-FOR1048. T.G. thanks Gregory Falkovich for the support. The authors acknowledge Patrick Teubner for the implementation of wavelet compression, and thank Tobias Schäfer for helpful discussions.

\section{References}

[1] CUDA toolkit documentation, 2014. http://docs.nvidia.com/cuda/index.html.

[2] Michael Assaf, Elijah Roberts, and Zaida Luthey-Schulten. Determining the stability of genetic switches: Explicitly accounting for mrna noise. Phys. Rev. Lett., 106:248102, 2011.

[3] David Backus, Mikhail Chernov, and Ian Martin. Disasters implied by equity index options. The Journal of Finance, 66:1969-2012, 2011.

[4] E. Balkovsky, G. Falkovich, I. Kolokolov, and V. Lebedev. Intermittency of Burgers' turbulence. Phys. Rev. Lett., 78:1452, 1997.

[5] Robert J. Barro. Rare disasters and asset markets in the twentieth century. Quarterly Journal of Economics, 2006.

[6] Antonio Celani, Massimo Cencini, and Alain Noullez. Going forth and back in time: a fast and parsimonious algorithm for mixed initial/final-value problems. Physica D: Nonlinear Phenomena, 195(3):283-291, 2004.

[7] A. I. Chernykh and M. G. Stepanov. Large negative velocity gradients in Burgers turbulence. Phys. Rev. E, 64:026306, 2001.

[8] C. de Dominicis. Techniques de renormalisation de la théorie des champs et dynamique des phénomènes critiques. J. Phys. C, 1:247, 1976.

[9] Amir Dembo and Ofer Zeitouni. Large deviations techniques and applications. SpringerVerlag, Berlin, 2010.

[10] W. E, W. Ren, and E. Vanden-Eijnden. Minimum action method for the study of rare events. Commun. Pure Appl. Math., 57:1-20, 2004.

[11] Weinan E, Weiqing Ren, and Eric Vanden-Eijnden. String method for the study of rare events. Phys. Rev. B, 66(5):052301, 2002.

[12] Vlad Elgart and Alex Kamenev. Classification of phase transitions in reaction-diffusion models. Phys. Rev. E, 74:041101, 2006.

[13] H. C. Fogedby and W. Ren. Minimum action method for the Kardar-Parisi-Zhang equation. Phys. Rev. E, 80:041116, 2009.

[14] Mark I Freidlin and Alexander D Wentzell. Random perturbations of dynamical systems, volume 260. Springer, 2012.

[15] T. Grafke, R. Grauer, and T. Schäfer. Instanton filtering for the stochastic Burgers equation. J. Phys. A, 46(6):62002, 2013.

[16] T. Grafke, R. Grauer, T. Schäfer, and E. Vanden-Eijnden. Relevance of instantons in Burgers turbulence. arXiv preprint arXiv:1412.0255, December 2014. 
[17] T. Grafke, R. Grauer, T. Schäfer, and Eric Vanden-Eijnden. Arclength parametrized Hamilton's equations for the calculation of instantons. Multiscale Modeling E Simulation, 12(2):566$580,2014$.

[18] V. Gurarie and A. Migdal. Instantons in the Burgers equation. Phys. Rev. E, 54:4908, 1996.

[19] Graeme Henkelman and Hannes Jónsson. Improved tangent estimate in the nudged elastic band method for finding minimum energy paths and saddle points. The Journal of Chemical Physics, 113:9978, 2000.

[20] M. Heymann and E. Vanden-Eijnden. Pathways of maximum likelihood for rare events in nonequilibrium systems: application to nucleation in the presence of shear. Phys. Rev. Lett., 100(14):140601, 2008.

[21] M. Heymann and E. Vanden-Ejnden. The geometric minimum action method: A least action principle on the space of curves. Commun. Pure Appl. Math., 61:1053, 2008.

[22] H.K. Janssen. On a Lagrangian for classical field dynamics and renormalization group calculations of dynamical critical properties. Z. Physik B, 23:377, 1976.

[23] H. A. Kramers. Brownian motion in a field of force and the diffusion model of chemical reactions. Physica, 7(4):284-304, April 1940.

[24] Jason Laurie and Freddy Bouchet. Computation of rare transitions in the barotropic quasigeostrophic equations. arXiv preprint arXiv:1409.3219, 2014.

[25] P. C. Martin, E. D. Siggia, and H. A. Rose. Statistical dynamics of classical systems. Phys. Rev. A, 8:423, 1973.

[26] M. Onorato, S. Residori, U. Bortolozzo, A. Montina, and F.T. Arecchi. Rogue waves and their generating mechanisms in different physical contexts. Physics Reports, 528(2):47 - 89, 2013.

[27] Maurice J. Schmeits and Henk A. Dijkstra. Bimodal behavior of the Kuroshio and the Gulf Stream. J. Phys. Ocean., 31:3435, 2001.

[28] Eric Vanden-Eijnden and Matthias Heymann. The geometric minimum action method for computing minimum energy paths. The Journal of chemical physics, 128:061103, 2008.

[29] E. Vanden-Ejnden and M. Heymann. The geometric minimum action method for computing minimum energy paths. Jour. Chem. Phys., 128:061103, 2008.

[30] Q. Wang, P. Moin, and G. Iaccarino. Minimal Repetition Dynamic Checkpointing Algorithm for Unsteady Adjoint Calculation. SIAM Journal on Scientific Computing, 31(4):2549-2567, January 2009.

[31] Xiang Zhou, Weiqing Ren, and Weinan E. Adaptive minimum action method for the study of rare events. J. Chem. Phys., 128:104111, 2008. 